This is a non peer-reviewed preprint submitted to EarthArxiv and is in revision in Geophysical Research Letters

\title{
Analog forecasting of extreme-causing weather patterns using deep learning
}

\author{
Ashesh Chattopadhyay ${ }^{1}$, Ebrahim Nabizadeh ${ }^{1}$, and Pedram \\ Hassanzadeh ${ }^{1,2, *}$ \\ ${ }^{1}$ Department of Mechanical Engineering, Rice University \\ ${ }^{2}$ Department of Earth, Environmental and Planetary Sciences, \\ Rice University \\ ${ }^{*}$ Corresponding author: pedram@rice.edu
}

July 26, 2019

\begin{abstract}
Numerical weather prediction (NWP) models require ever-growing computing time/resources, but still, have difficulties with predicting weather extremes. Here we introduce a data-driven framework that is based on analog forecasting (prediction using past similar patterns) and employs a novel deep learning pattern-recognition technique (capsule neural networks, CapsNets) and impact-based auto-labeling strategy. CapsNets are trained on mid-tropospheric large-scale circulation patterns (Z500) labeled $0-4$ depending on the existence and geographical region of surface temperature extremes over North America several days ahead. The trained networks predict the occurrence/region of cold or heat waves, only using Z500, with accuracies (recalls) of $69 \%-45 \%(77 \%-48 \%)$ or $62 \%-41 \%$ $(73 \%-47 \%) 1-5$ days ahead. CapsNets outperform simpler techniques such as convolutional neural networks and logistic regression. Using both temperature and Z500, accuracies (recalls) with CapsNets increase to $\sim 80 \%$ (88\%), showing the promises of multi-modal data-driven frameworks for accurate/fast extreme weather predictions, which can augment NWP efforts in providing early warnings.
\end{abstract}

\section{Introduction}

Predicting extreme weather events such as heat waves and cold spells is of significant scientific and societal importance. However, despite decades of progress in weather prediction, mostly through improving computationally-demanding numerical weather prediction (NWP) models and data-assimilation techniques $[1,2]$, forecasting the anomalous atmospheric circulation patterns that often 
This is a non peer-reviewed preprint submitted to EarthArxiv and is in revision in Geophysical Research Letters

drive these extreme events has remained a challenge. For example, blocking events, which are large-scale, persistent, high-pressure systems that block/divert the usual eastward winds [3], have caused some of the most devastating natural disasters in recent times such as the 2003 and 2010 heat waves in Europe [4, 3]. Yet, the state-of-the-art NWP models have difficulties with accurately predicting the formation and persistence of blocking events $[5,6,7]$. Overall, the key characteristics of extreme-causing weather patterns, their dynamics, and conditions that lead to their formation (i.e., precursors) are not well understood $[3,8,9,10,11,12,13]$.

Recent advances in artificial intelligence have revolutionized how problems in various domains of business and science are approached [14, 15]. For example, in climate science, using machine learning techniques to accurately and efficiently represent unresolved physical processes in the atmosphere and ocean has produced promising results $[16,17,18,19,20]$, and has the potential to significantly improve climate modeling and long-term climate projections in the coming years $[21,22,23]$. Moreover, deep learning techniques have been very successful in predicting some types of sequential data [15]. Consequently, whether such techniques can be used for data-driven forecasting of the spatiotemporal evolution of the weather systems (and their extreme events), e.g., after training on NWP model outputs or observational data, has become an active area of research. However, recent efforts pursuing this approach, which essentially requires a neural network to accurately, for some time, emulate the high-dimensional nonlinear dynamics governing the evolution of the turbulent atmospheric circulation, have produced mixed results even in overly simplified models of atmosphere [24, 25, 26].

In the current study, for data-driven prediction of extreme-causing weather patterns, we introduce an alternative framework that is based on analog forecasting, i.e., making prediction by finding similar pattern(s), or analog(s), in the past $[27,28]$. In the historical context, before the advent of powerful electronic computers and stable numerical schemes for integrating the partial differential equations of the NWP models, analog forecasting was a key tool in weather prediction; e.g., it was used in the planning of the D-Day for the 1944 Normandy invasion [29]. Analog forecasting was used less frequently in later decades, due to the challenges in finding useful analogs and the rapid growth of NWP [28], although the approach has the potential for a comeback given the rapid increase in data and emergence of new auxiliary methods [29,30].

Here, we build our data-driven framework on analog forecasting because the patterns of the circulation, e.g., the relative positions of high- and low-pressure systems, play a key role in the spatio-temporal evolution of the circulation and the initiation of extreme events at the surface, and analog forecasting essentially casts weather prediction as a complex pattern-recognition problem, an area that has been truly revolutionized by deep learning in recent years [14, 15]. Rather than looking for one perfect analog or a combination of near-perfect analogs, as pursued in traditional analog forecasting [28], our framework employs deep learning techniques to decipher the complex relationship between the weather patterns (at the altitude of $\sim 5 \mathrm{~km}$ ) and the extreme events (at the surface) 
This is a non peer-reviewed preprint submitted to EarthArxiv and is in revision in Geophysical Research Letters

among all training samples. The purpose of this paper is to provide a proof-ofconcept for this framework.

\section{Materials and Methods}

\subsection{Data}

We use daily data from the Large-Ensemble (LENS) Community Project [31], which consists of a 40-member ensemble of fully-coupled atmosphere-oceanland-ice simulations with 1920 - 2005 historical radiative forcing. To ensure abundant training samples for the purpose of demonstrating a proof-of-concept for the framework, we have chosen to use data from a multi-ensemble climate model, rather than reanalysis data (see Discussion). Still, the simulated atmospheric circulation is non-stationary, turbulent, and multi-scale, with complexities similar to those of the real atmosphere, thus providing a challenging testbed for our data-driven extreme weather prediction framework.

Extreme hot (cold) events are defined as the anomalous land surface temperature, T2m hereafter, in the 99 (1) percentile that persist for at least 5 consecutive days in summer (winter) over North America between $30^{\circ} \mathrm{N}-60^{\circ} \mathrm{N}$. The onsets of extreme events are clusteredx into 4 distinct yet cohesive geographical regions, corresponding to Northern Canada, Southern US, Western US-Canada, and Eastern US-Canada in summers (Figure1). See the supporting information for more details on definition of extreme events and the clustering analysis. Our objective here is to predict, a few days ahead, the occurrence of an extreme event and its location, in terms of the 4 clusters, from just knowing a single variable, geopotential height at $500 \mathrm{mb}$ (Z500 hereafter).

\subsection{Deep Learning Techniques}

We use two state-of-the-art deep learning techniques for pattern recognition: convolutional neural network (ConvNet or CNN) $[14,15]$ and a more advanced method, capsule neural network (CapsNet) [32]. The key advantage of both methods over traditional image-processing techniques is that the filters used for feature extraction are learned for each dataset through an algorithm called backpropagation [15], rather than being hand-engineered and specified beforehand. ConvNet is the groundbreaking method that has transformed image processing since 2011, but because of a property called equivariance that is discussed later, CapsNet is expected to work even better for our framework.

Both methods are supervised, meaning that they have to be first trained on labeled patterns. However, given the incomplete understanding of extremecausing weather patterns and their complexities, expert-labeled data will not be useful for our objective. For example, indices designed to find blocking patterns in the Z500 field based on their presumed properties are known to perform poorly, e.g., in identifying extreme-causing patterns even on the same day as the heat or cold extreme events [33]. To overcome this challenge, here we de- 
This is a non peer-reviewed preprint submitted to EarthArxiv and is in revision in Geophysical Research Letters

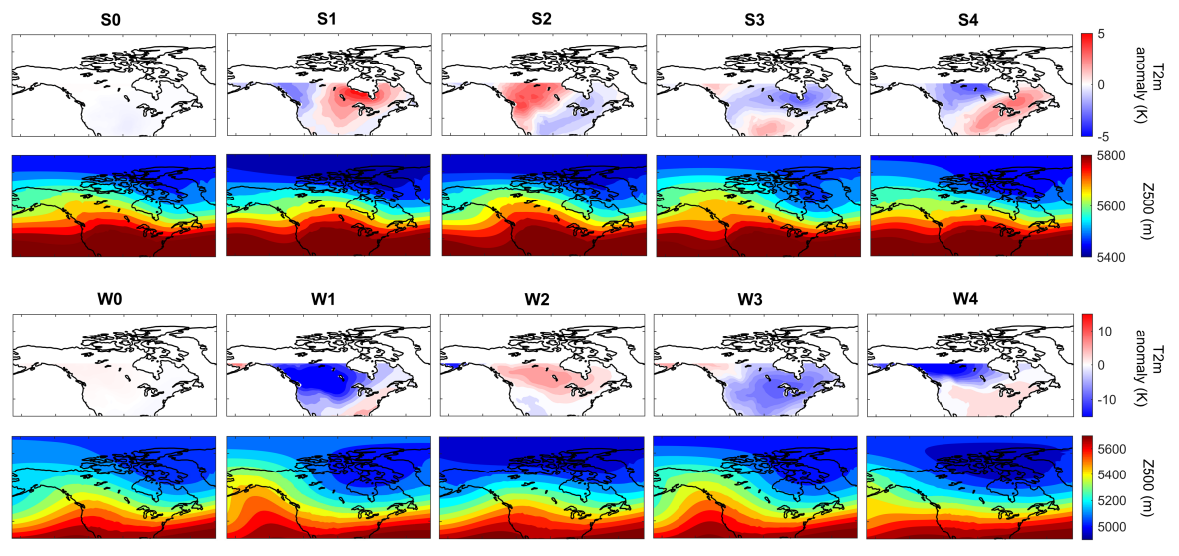

Figure 1: Cluster centers of T2m anomalies at the onsets and Z500 patterns of 3 days earlier. The top (bottom) two rows correspond to summers (winters). S0 (W0) shows the average of T2m and Z500 patterns from days with no heat wave (cold spell). S1-S4 and W1-W4 are obtained from K-means clustering the anomalous T2m patterns at onsets into four classes, which roughly separates the extreme events into four geographical regions: Northern Canada (S1), Western US-Canada (S2), Southern US (S3), and Eastern US-Canada (S4) in summers, and North-West US-Canada (W1), Alaska (W2), North-East US-Canada (W3), and Northern Canada (W4) in winters. Rows 1 and 3 show the cluster centers while rows 2 and 4 show the average of Z500 patterns 3 days before the onsets for each cluster. See the supporting information for more details.

vise an impact-based auto-labeling strategy: knowing the surface temperature over North America on a given day, the Z500 pattern of several days earlier is labeled as 0 (no extreme onset) or 1, 2, 3 or 4 (the cluster indices of T2m extremes). We highlight that in conventional deep learning applications, labeling and training/testing are all conducted on the same feature map; however, in the impact-based labeling strategy introduced here, we label based on differences in one feature map (T2m) but train/test on another feature map (Z500), in order to predict the original feature map $(\mathrm{T} 2 \mathrm{~m})$. While more challenging, the impactbased auto-labeling strategy circumvents the need for a full understanding of the complex and nonlinear relationship between the predictor (Z500) and the impact of interest $(\mathrm{T} 2 \mathrm{~m})$.

\subsection{Data-driven Extreme Weather Prediction Framework}

The schematic of the entire data-driven prediction framework is shown in Figure 2. The CapsNet (or ConvNet) is first trained on daily Z500 patterns that are auto-labeled using the above strategy. Then, for a never-seen-before Z500 pattern, the trained network can predict whether a surface temperature extreme event will occur in North America a few days ahead, and if so, in which of the 
This is a non peer-reviewed preprint submitted to EarthArxiv and is in revision in Geophysical Research Letters

four geographical regions. To further show the promises of this framework in predicting extreme weather events, results with training/testing on inputs that consist of both Z500 and T2m are presented as well.

\subsection{Skill Metrics: Accuracy and Recall}

We report the prediction skills in terms of total accuracy of the testing set, computed as the number of test samples from all 5 clusters whose cluster index is correctly predicted divided by the total number of test samples, and recall, computed as the number of test samples from the four clusters with extreme events $(1-4)$ whose cluster index is correctly predicted divided by the total number of test samples in clusters $1-4$. We computed the recall because for extreme weather prediction, missed events are much more undesirable than false alarms. Together, accuracy and recall fully quantify the skills of the framework for a multi-class prediction. The accuracy for individual clusters, computed as the number of correctly predicted test samples from that cluster divided by the total number of test samples from that cluster, is the receiver operating characteristic (ROC) score, a common forecast skill metric [34, 12].

\section{Results}

Figure 3 shows the performance of CapsNet for predicting cold spells and heat waves using the Z500 patterns from $1-5$ days earlier. The accuracies for lead times of 1 to 5 days are between $68.8 \% \pm 0.3 \%$ and $45.1 \% \pm 0.1 \%$ in winter, and $61.6 \% \pm 0.0 \%$ and $40.6 \% \pm 0.1 \%$ in summer, against a $20 \%$ random chance in a 5 -class prediction. The recalls are consistently higher, between $77.2 \% \pm 0.3 \%$ and $48.1 \% \pm 0.1 \%$ in winter and $72.8 \% \pm 0.1 \%$ and $46.6 \% \pm 0.1 \%$ in summer. Examining the prediction accuracy for individual clusters shows that the inaccuracies largely result from false alarms due to non-extreme-events (cluster 0) incorrectly predicted as an extreme event somewhere in North America (clusters $1-4)$. False alarms can be reduced by adding more constraints on Z500 during labeling, e.g., requiring daily Z500 anomalies to exceed 1.5 standard deviation (SD); however, we have chosen to avoid subjective criteria and have only used the impact (i.e., T2m extreme) for labeling. Furthermore, we have focused on minimally pre-processed inputs, e.g., we have not de-trended Z500 patterns and instead have used the full Z500 patterns (see the supporting information), which are non-stationary due to low-frequency coupled atmosphere-ocean modes of climate variability and changes in the radiative forcing from $1920-2005$.

The results in Figures 3(a)-(b) are obtained with a training set containing $N=750$ samples from each of the 5 clusters. Figures 3(c)-(d) show that as the size of the training set is reduced, the accuracies for winter barely decline. Even when the number of training samples per cluster is reduced almost by a factor of 4 to 187 or 168 (depending on the lag), the largest decrease in accuracy is $4.7 \%$ (for day -4). In summer, the effect of the size of the training set is more pronounced especially at longer lead times, e.g., the accuracy for 5-day 
This is a non peer-reviewed preprint submitted to EarthArxiv and is in revision in Geophysical Research Letters

prediction declines by $9.5 \%$ when the training set is reduced by a factor of 4 . Overall, the weak dependence of the accuracy on the size of the training set is encouraging for practical purposes (see Discussion), but it also suggests that likely, higher accuracies could not be achieved even if we had more training samples (see below).

The prediction skills in summers are lower than those in winters. Figure 1 shows that the Z500 patterns corresponding to different clusters are much more similar in summers than in winters, suggesting that it should be harder to identify the correct cluster of a pattern in summer. Still, that CapsNet can differentiate between patterns that have such similar averages (i.e., cluster centers) with the accuracy (recall) of $48.2 \% \pm 0.1 \%(55.6 \% \pm 0.1 \%)$ shows the effectiveness of the framework. Furthermore, dynamics of heat waves are more complex than cold spells and the mid-tropospheric circulation patterns (the only predictor here) are not the only driver: cold spells are mostly due to equatorward advection of cold air from higher latitudes while the heat waves are caused by a combination of horizontal advection and adiabatic and clear-sky radiative warmings [35, 36, 37]. Moreover, land-atmosphere feedbacks play a role in the dynamics of heat waves [38].

The results of Figure 3 show the power of our data-driven framework for predicting the surface temperature extreme events using a single variable (Z500) that represents mid-tropospheric circulation, i.e., predicting extreme-causing weather patterns. The above discussions on the weak dependence of accuracy on the size of the training set and the dynamics of the extreme temperature events suggest that including more variables as the predictor and pursuing a multi-modal framework would lead to better prediction skills for the extreme temperature events, particularly at longer lead times. It should be highlighted that even for winters, where meridional advection dominates, including information from other altitudes of troposphere and stratosphere (e.g., to account for polar vortex variability) are expected to improve the prediction skills (see Discussion).

To demonstrate the promises of such multi-modal data-driven frameworks, we have repeated the analysis of Figure 3 but by inputting the patterns of Z500 and anomalous $\mathrm{T} 2 \mathrm{~m}$ together into CapsNet in the training and testing phases. Figure 4 shows that the accuracies (recalls) for lead times of 1 to 5 days rise to between $82.0 \% \pm 1.5 \%(87.8 \% \pm 1.4 \%)$ and $76.7 \% \pm 2.5 \%(88.2 \% \pm 2.3 \%)$ in winter and $79.3 \% \pm 1.6 \%(87.2 \% \pm 1.7 \%)$ and $75.8 \% \pm 2.7 \%(87.2 \% \pm 2.6 \%)$ in summer, significantly improving the prediction skills, particularly in the longer lead times. With T2m included, the false alarms decline in most cases, and the accuracies/recalls hardly change with lead time or size of the training set. It should be highlighted that the high prediction skills with Z500+T2m are not simply due to the temporal memory as a result of including $\mathrm{T} 2 \mathrm{~m}$ of earlier days. With $N=750$, training and testing the CapsNets on T2m alone result in accuracies that are consistently lower, between $0.6 \%-5.2 \%(1.5 \%-4.5 \%)$, than the accuracies with Z500+T2m in winter (summer), showing that information about the atmospheric circulation adds to the predictive skills.

The accurate and robust 1 to 5 -day predictions in Figure 4 suggest that the 
This is a non peer-reviewed preprint submitted to EarthArxiv and is in revision in Geophysical Research Letters

multi-modal framework using Z500+T2m, or even more variables (see Discussion), might have high prediction skills for lead times beyond 5 days. However, such longer predictions will require using Z500 patterns (and some of the other variables) at the planetary-scales, which, for the best performance of the framework, needs CapsNet (and ConvNet) architectures capable of accounting for the Earth's spherical geometry, e.g., the zonal periodicity and decrease of area with latitude. Extending the framework to planetary-scales and longer prediction lead times is left for future work, which will benefit from recent advances in developing spherical ConvNets [39, 40].

We have also conducted the analyses in Figures 3 and 4 with CapsNet replaced with two simpler methods: i) ConvNet, which is a deep learning method of growing interest in climate science (and other disciplines) and was used, for example, in the pioneering work of Liu et. al., [41] to identify tropical cyclones and atmospheric rivers, and ii) logistic regression [15], which is a widely-used machine learning method that has been employed in some past weather forecasting efforts $[42,43,44]$. Figures S1 and S2 show that the CapsNets consistently outperform the ConvNets (except for one Case: 4-day lead time in summer). For predictions with Z500 (Z500+T2m), the accuracies of CapsNets are, on average, higher than ConvNets by $2.8 \%(7.7 \%)$ in winters and $0.7 \%(7.1 \%)$ in summers. Furthermore, as the size of the training set is reduced, the accuracy of ConvNets degrades more than that of CapsNet, particularly in the multi-modal approach with Z500+T2m (Figures S1-S2(c)-(d)). Due to their different architectures (see supporting information), CapsNets extract more features and information from each pattern compared to ConvNets, and are thus expected to work well even with relatively small training sets. More importantly, CapsNets account for the relative position of features (a property called equivariance) [32]. Relative positions of features in climate data are important, e.g., high-pressure systems on the poleward side of low-pressure systems might stall and cause weather extremes, while low-pressure systems on the poleward side of high-pressure system often move eastward without causing extreme events.

The accuracy of logistic regression is consistently lower than that of ConvNets (and thus CapsNet), see Figures S1-S2. For predictions with Z500, the accuracies of CapsNets are, on average, higher than those of logistic regression by $11.4 \%(19.6 \%)$ in winters (summers). These results show the advantage of more advanced deep learning techniques over simpler ones such as ConvNet and logistic regression, and suggest that future studies in climate and environmental sciences might benefit from using CapsNets (and might benefit even more from deep learning techniques designed specifically for multi-scale, spatio-temporal, chaotic data).

Note that we have not compared the performance of our framework with persistence or climatology, which are two common baseline methods [45], because they could not be formulated to predict T2m extremes based on inputs of Z500 patterns, and that by definition, there is no T2m extreme within 5 days of the onsets (see supporting information). 
This is a non peer-reviewed preprint submitted to EarthArxiv and is in revision in Geophysical Research Letters

\section{Discussion}

The results of Figure 3 show the skills of the data-driven framework in predicting high-impact events (e.g., T2m extremes) only through limited information about the events's driver (or one of the key drivers), i.e., Z500 patterns in this case, and without any information about the impact itself. This skillful prediction of extreme-causing weather patterns provides a proof-of-concept for the framework (Figure 2). We emphasize that the key components of this datadriven framework are the novel impact-based auto-labeling technique and the power of CapsNets in pattern recognition, which together enable the framework to decipher the relationship between the T2m and Z500 patterns, and the temporal evolution of Z500 patterns, despite challenges such as sensitivity of nonlinear systems to small perturbations in initial conditions [27].

Based on the results of Figure 4, the multi-modal framework, once equipped with spherical CapsNets for planetary-scale inputs, may offer a promising datadriven approach to prediction. Higher accuracies and longer prediction lead times (at the weekly to seasonal time-scales, which are of the most utility and interest) might be achievable by including variables such as geopotential heights at more tropospheric levels, soil moisture, and outgoing longwave radiation, as well as information from the slow-varying boundaries of the troposphere such as tropical and extratropical sea-surface temperature (e.g., from Pacific Ocean), tropical atmosphere (e.g., Madden-Julien Oscillation), sea ice, and stratosphere, which are all known to enhance predictive skills for the midlatitude extreme events $[12,46,47]$.

The data-driven extreme event prediction framework introduced here can be useful in (at least) two ways: 1) to provide early warnings of extreme events and guide the public and NWP efforts, and 2) to identify the precursors of extreme events using ideas from interpretable machine learning. Regarding the former, one of the most appealing and powerful aspects of a data-driven framework is the possibility of training on observational data. Here, the main challenges in using observed climate data for training are that such records are short, and the data are non-stationary in time. Reanalysis products are available for as early as 1850, although the data from before 1979 are derived from very limited direct observations. The LENS data used in this study have complexity and non-stationarity similar to that of the reanalysis data, however, the 40-member ensemble simulations provide, e.g., $\sim 300000$ days of data in winters, which is much larger than what can be obtained from reanalysis datasets. Given our focus on the onsets of extreme events in the 1 or 99 percentile, from the LENS data, we only used as high as 750 and as low as 168 samples per cluster for training. Figures 3 and 4 show that even with the smallest training set, skillful multi-class predictions are obtained. Furthermore, synthetic datasets that are based on observational data, already produced using statistical resampling [48] or can be produced using advanced deep learning methods such as generative adversarial networks [15], can provide additional data for training. The above discussion suggests that it might be possible to use data derived from observations (and not NWP or climate models) for training of the data-driven 
This is a non peer-reviewed preprint submitted to EarthArxiv and is in revision in Geophysical Research Letters

framework. We highlight again that the purpose of this paper is to provide a proof-of-concept for the framework. Evaluating the performance of the framework trained on reanalysis data, and comparing the forecast skills with those of the NWP models, are admittedly the essential and important next steps, and are currently underway.

Data from large-ensemble, high-resolution NWP model simulations can also be used for training. The very high-resolution NWP models, which need prohibitive computing time/resources to run continuously, simulate the atmospheric circulation, and in particular extreme-causing patterns, with higher fidelity compared to the simulations with lower resolutions [2, 49]. The advantage of using the data-driven framework, trained on high-resolution NWP models, is that it can yield extremely fast and inexpensive regional predictions, which can provide early warnings and guide the deployment of computing and sensing resources for large-ensemble, high-resolution NWP of a region predicted to experience an extreme event in several days (or longer).

In this study we conducted 5-class predictions based on extreme events over North America clustered (using K-means algorithm [50]) into 4 geographical regions. However, other clustering algorithms, number of clusters etc. could be used (see supporting information), or alternatively, separate data-driven frameworks can be developed for binary (yes/no) extreme predictions in each region of interest, e.g., around Greater Houston, Bay Area, Greater Boston. Understanding which of the approaches discussed above (differing in training data and framework design) lead to the best data-driven prediction skills and better handle practical limitations requires extensive research and should be addressed in future studies.

Precursors of extreme-causing weather patterns such as blocking events are not well understood $[3,11,13]$ and identifying them can lead to a better dynamical understanding of extreme events and potentially improving weather and climate models. Given that CapsNets show skills in predicting the extremecausing weather patterns, it is of interest to understand how the neural network has "learned" what key features to look for. However, understanding how deep neural networks work is known to be challenging and an active area of research. In future work, the feature maps and filters should be examined to seek an understanding of how CapsNets differentiate between patterns that in a few days lead to different $\mathrm{T} 2 \mathrm{~m}$ clusters. Investigating the first convolutional layer can be informative [20], but given that the learning is believed to happen in the deeper layers where the filters are acting on reduced feature maps to identify the key features, recent advances in interpretable deep learning [51] should be employed to explain how the Z500 patterns have been differentiated.

Finally, our data-driven framework can be readily generalized for prediction of other high-impact climatic and environmental phenomena, e.g., extreme precipitation or severe air pollution events, just to name a few. The impact-based auto-labeling strategy circumvents the need for a full understanding of the relationship between the impact of interest and its driver(s). Needless to say, domain expertise is still critical in designing the auto-labeling strategy, e.g., in choosing the relevant variables and spatio-temporal scales. 
This is a non peer-reviewed preprint submitted to EarthArxiv and is in revision in Geophysical Research Letters

Acknowledgment: We thank Paige Bailey, Ashkan Borna, Packard Chan, Zhiming Kuang, Karen McKinnon, Krishna Palem, Laura Schaefer, Devika Subramanian, and Chris Wikle for insightful discussions. Computational resources were provided by the NSF XSEDE allocation ATM170020. This work was supported by NASA grant 80NSSC17K0266 and an Early-Career Research Fellowship from the Gulf Research Program of the National Academies of Science, Engineering, and Medicine (to P.H.). A.C. thanks the Rice University Ken Kennedy Institute for a BP HPC Graduate Fellowship. The LENS dataset is available from http://www.cesm.ucar.edu/projects/community-projects/ LENS/. The CapsNet and ConvNet codes are publicly available on GitHub: https://github.com/ashesh6810/DLC_extreme.

\section{References}

[1] Peter Bauer, Alan Thorpe, and Gilbert Brunet. The quiet revolution of numerical weather prediction. Nature, 525(7567):47, 2015.

[2] Richard B Alley, Kerry A Emanuel, and Fuqing Zhang. Advances in weather prediction. Science, 363(6425):342-344, 2019.

[3] Tim Woollings, David Barriopedro, John Methven, Seok-Woo Son, Olivia Martius, Ben Harvey, Jana Sillmann, Anthony R Lupo, and Sonia Seneviratne. Blocking and its response to climate change. Current Climate Change Reports, 4(3):287-300, 2018.

[4] David Barriopedro, Erich M Fischer, Jürg Luterbacher, Ricardo M Trigo, and Ricardo García-Herrera. The hot summer of 2010: redrawing the temperature record map of europe. Science, 332(6026):220-224, 2011.

[5] Laura Ferranti, Susanna Corti, and Martin Janousek. Flow-dependent verification of the ecmwf ensemble over the euro-atlantic sector. Quarterly Journal of the Royal Meteorological Society, 141(688):916-924, 2015.

[6] Mio Matsueda. Predictability of euro-russian blocking in summer of 2010. Geophysical Research Letters, 38(6), 2011.

[7] Joanna L Pelly and Brian J Hoskins. How well does the ecmwf ensemble prediction system predict blocking? Quarterly Journal of the Royal Meteorological Society: A journal of the atmospheric sciences, applied meteorology and physical oceanography, 129(590):1683-1702, 2003.

[8] Haiyan Teng, Grant Branstator, Hailan Wang, Gerald A Meehl, and Warren M Washington. Probability of us heat waves affected by a subseasonal planetary wave pattern. Nature Geoscience, 6(12):1056, 2013.

[9] Dim Coumou, Jascha Lehmann, and Johanna Beckmann. The weakening summer circulation in the northern hemisphere mid-latitudes. Science, 348(6232):324-327, 2015. 
This is a non peer-reviewed preprint submitted to EarthArxiv and is in revision in Geophysical Research Letters

[10] Daniel E Horton, Nathaniel C Johnson, Deepti Singh, Daniel L Swain, Bala Rajaratnam, and Noah S Diffenbaugh. Contribution of changes in atmospheric circulation patterns to extreme temperature trends. Nature, 522(7557):465, 2015.

[11] Pedram Hassanzadeh and Zhiming Kuang. Blocking variability: Arctic amplification versus arctic oscillation. Geophysical Research Letters, 42(20):8586-8595, 2015.

[12] Karen Aline McKinnon, A Rhines, MP Tingley, and P Huybers. Longlead predictions of eastern united states hot days from pacific sea surface temperatures. Nature Geoscience, 9(5):389, 2016.

[13] Noboru Nakamura and Clare SY Huang. Atmospheric blocking as a traffic jam in the jet stream. Science, 361(6397):42-47, 2018.

[14] Yann LeCun, Yoshua Bengio, and Geoffrey Hinton. Deep learning. nature, 521(7553):436, 2015.

[15] Ian Goodfellow, Yoshua Bengio, and Aaron Courville. Deep learning. MIT press, 2016.

[16] Stephan Rasp, Michael S Pritchard, and Pierre Gentine. Deep learning to represent subgrid processes in climate models. Proceedings of the National Academy of Sciences, 115(39):9684-9689, 2018.

[17] Noah D Brenowitz and Christopher S Bretherton. Prognostic validation of a neural network unified physics parameterization. Geophysical Research Letters, 45(12):6289-6298, 2018.

[18] Paul A O'Gorman and John G Dwyer. Using machine learning to parameterize moist convection: Potential for modeling of climate, climate change, and extreme events. Journal of Advances in Modeling Earth Systems, 10(10):2548-2563, 2018.

[19] Thomas Bolton and Laure Zanna. Applications of deep learning to ocean data inference and subgrid parameterization. Journal of Advances in Modeling Earth Systems, 11(1):376-399, 2019.

[20] Hesam Salehipour and W Richard Peltier. Deep learning of mixing by two atoms of stratified turbulence. Journal of Fluid Mechanics, 861, 2019.

[21] Tapio Schneider, Shiwei Lan, Andrew Stuart, and João Teixeira. Earth system modeling 2.0: A blueprint for models that learn from observations and targeted high-resolution simulations. Geophysical Research Letters, 44(24), 2017.

[22] Pierre Gentine, Mike Pritchard, Stephan Rasp, Gael Reinaudi, and Galen Yacalis. Could machine learning break the convection parameterization deadlock? Geophysical Research Letters, 45(11):5742-5751, 2018. 
This is a non peer-reviewed preprint submitted to EarthArxiv and is in revision in Geophysical Research Letters

[23] Markus Reichstein, Gustau Camps-Valls, Bjorn Stevens, Martin Jung, Joachim Denzler, Nuno Carvalhais, and Prabhat. Deep learning and process understanding for data-driven Earth system science. Nature, 566(7743):195, 2019.

[24] Peter D Dueben and Peter Bauer. Challenges and design choices for global weather and climate models based on machine learning. Geoscientific Model Development, 11(10):3999-4009, 2018.

[25] Pantelis R Vlachas, Wonmin Byeon, Zhong Y Wan, Themistoklis P Sapsis, and Petros Koumoutsakos. Data-driven forecasting of high-dimensional chaotic systems with long short-term memory networks. Proceedings of the Royal Society A: Mathematical, Physical and Engineering Sciences, 474(2213):20170844, 2018.

[26] Sebastian Scher. Toward data-driven weather and climate forecasting: Approximating a simple general circulation model with deep learning. Geophysical Research Letters, 45(22):12-616, 2018.

[27] Edward N Lorenz. Atmospheric predictability as revealed by naturally occurring analogues. Journal of the Atmospheric sciences, 26(4):636-646, 1969 .

[28] Huug Van den Dool. Empirical methods in short-term climate prediction. Oxford University Press, 2007.

[29] Patrick L McDermott and Christopher K Wikle. A model-based approach for analog spatio-temporal dynamic forecasting. Environmetrics, 27(2):70$82,2016$.

[30] Zhizhen Zhao and Dimitrios Giannakis. Analog forecasting with dynamicsadapted kernels. Nonlinearity, 29(9):2888, 2016.

[31] JE Kay, C Deser, A Phillips, A Mai, C Hannay, G Strand, JM Arblaster, SC Bates, G Danabasoglu, J Edwards, M Holland, P Kushner, J.-F. Lamarque, D. Lawrence, K Lindsay, A. Middleton, E Munoz, R. Neale, K. Oleson, L. Polvani, and M Vertenstein. The community earth system model (CESM) large ensemble project: A community resource for studying climate change in the presence of internal climate variability. Bulletin of the American Meteorological Society, 96(8):1333-1349, 2015.

[32] Sara Sabour, Nicholas Frosst, and Geoffrey E Hinton. Dynamic routing between capsules. In Advances in neural information processing systems, pages 3856-3866, 2017.

[33] Pak-Wah Chan, Pedram Hassanzadeh, and Zhiming Kuang. Evaluating indices of blocking anticyclones in terms of their linear relations with surface hot extremes. Geophysical Research Letters, 46, 2019. 
This is a non peer-reviewed preprint submitted to EarthArxiv and is in revision in Geophysical Research Letters

[34] Tom Fawcett. An introduction to ROC analysis. Pattern Recognition Letters, 27(8):861-874, 2006.

[35] Randall Dole, Martin Hoerling, Judith Perlwitz, Jon Eischeid, Philip Pegion, Tao Zhang, Xiao-Wei Quan, Taiyi Xu, and Donald Murray. Was there a basis for anticipating the 2010 russian heat wave? Geophysical Research Letters, 38(6), 2011.

[36] S Pfahl and H Wernli. Quantifying the relevance of atmospheric blocking for co-located temperature extremes in the northern hemisphere on (sub-) daily time scales. Geophysical Research Letters, 39(12), 2012.

[37] Tapio Schneider, Tobias Bischoff, and Hanna Płotka. Physics of changes in synoptic midlatitude temperature variability. Journal of Climate, 28(6):2312-2331, 2015.

[38] Diego G Miralles, Adriaan J Teuling, Chiel C Van Heerwaarden, and Jordi Vila-Guerau De Arellano. Mega-heatwave temperatures due to combined soil desiccation and atmospheric heat accumulation. Nature geoscience, 7(5):345, 2014.

[39] Taco S Cohen, Mario Geiger, Jonas Köhler, and Max Welling. Spherical cnns. arXiv preprint arXiv:1801.10130, 2018.

[40] Chiyu Jiang, Jingwei Huang, Karthik Kashinath, Philip Marcus, and Matthias Niessner. Spherical CNNs on unstructured grids. arXiv preprint arXiv:1901.02039, 2019.

[41] Yunjie Liu, Evan Racah, Joaquin Correa, Amir Khosrowshahi, David Lavers, Kenneth Kunkel, Michael Wehner, and William Collins. Application of deep convolutional neural networks for detecting extreme weather in climate datasets. arXiv preprint arXiv:1605.01156, 2016.

[42] Scott Applequist, Gregory E Gahrs, Richard L Pfeffer, and Xu-Feng Niu. Comparison of methodologies for probabilistic quantitative precipitation forecasting. Weather and Forecasting, 17(4):783-799, 2002.

[43] Gregory R Herman and Russ S Schumacher. Money doesn't grow on trees, but forecasts do: Forecasting extreme precipitation with random forests. Monthly Weather Review, 146(5):1571-1600, 2018.

[44] Kirien Whan and Maurice Schmeits. Comparing area probability forecasts of (extreme) local precipitation using parametric and machine learning statistical postprocessing methods. Monthly Weather Review, 146(11):3651$3673,2018$.

[45] Allan H Murphy. Climatology, persistence, and their linear combination as standards of reference in skill scores. Weather and Forecasting, 7(4):692698, 1992. 
This is a non peer-reviewed preprint submitted to EarthArxiv and is in revision in Geophysical Research Letters

[46] Mark P Baldwin and Timothy J Dunkerton. Stratospheric harbingers of anomalous weather regimes. Science, 294(5542):581-584, 2001.

[47] Bryan D Mundhenk, Elizabeth A Barnes, Eric D Maloney, and Cory F Baggett. Skillful empirical subseasonal prediction of landfalling atmospheric river activity using the madden-julian oscillation and quasi-biennial oscillation. npj Climate and Atmospheric Science, 1(1):7, 2018.

[48] Karen A McKinnon, Andrew Poppick, Etienne Dunn-Sigouin, and Clara Deser. An observational large ensemble uncertainty dueto compare observed and modeled temperature trend uncertainty due to internal variability. Journal of Climate, 30(19):7585-7598, 2017.

[49] Thomas Jung, MJ Miller, TN Palmer, P Towers, N Wedi, D Achuthavarier, JM Adams, EL Altshuler, BA Cash, JL Kinter Iii, L. Marx, C. Stan, and K. I. Hodges. High-resolution global climate simulations with the ECMWF model in Project Athena: Experimental design, model climate, and seasonal forecast skill. Journal of Climate, 25(9):3155-3172, 2012.

[50] Stuart Lloyd. Least squares quantization in PCM. IEEE Transactions on Information Theory, 28(2):129-137, 1982.

[51] Matthew D Zeiler and Rob Fergus. Visualizing and understanding convolutional networks. In European Conference on Computer Vision, pages 818-833. Springer, 2014. 
This is a non peer-reviewed preprint submitted to EarthArxiv and is in revision in Geophysical Research Letters

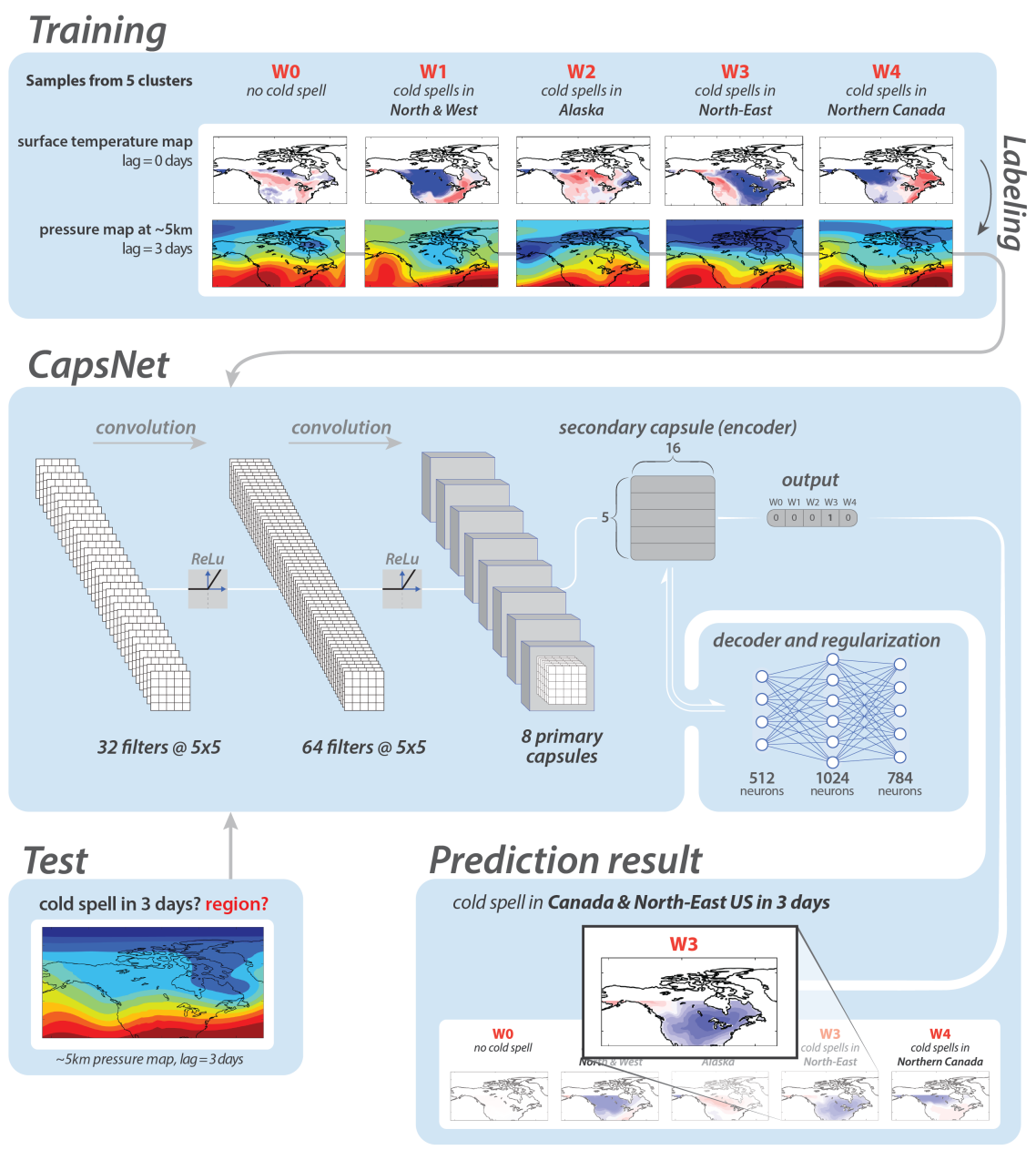

Figure 2: Schematic of the data-driven framework for prediction of cold spells based on Z500 patterns of 3 days earlier. Using the impact-based auto-labeling strategy, Z500 patterns are labeled W0, W1, W2, W3, or W4, depending on the cluster index of T2m three days ahead. The panels at the top show examples of T2m patterns at the onset and the corresponding Z500 patterns (from three days earlier) for each cluster. Only the Z500 patterns and their labels are inputted into the CapsNet during training. Once trained, the CapsNet can predict, from a given $\mathrm{Z} 500$ pattern, the $\mathrm{T} 2 \mathrm{~m}$ cluster index of three days later, thus predicting the occurrence and geographical region of cold spells. For the shown test example, a cold spell in W3 in 3 days is predicted. Note that for winters, Z500 patterns over a larger domain that extends across the Pacific Ocean to $145^{\circ} \mathrm{E}$ are inputted into the CapsNets, but a smaller domain is shown in this figure for better illustration (see supporting information). Separate CapsNets are trained for each season and each prediction lead time. 
This is a non peer-reviewed preprint submitted to EarthArxiv and is in revision in Geophysical Research Letters
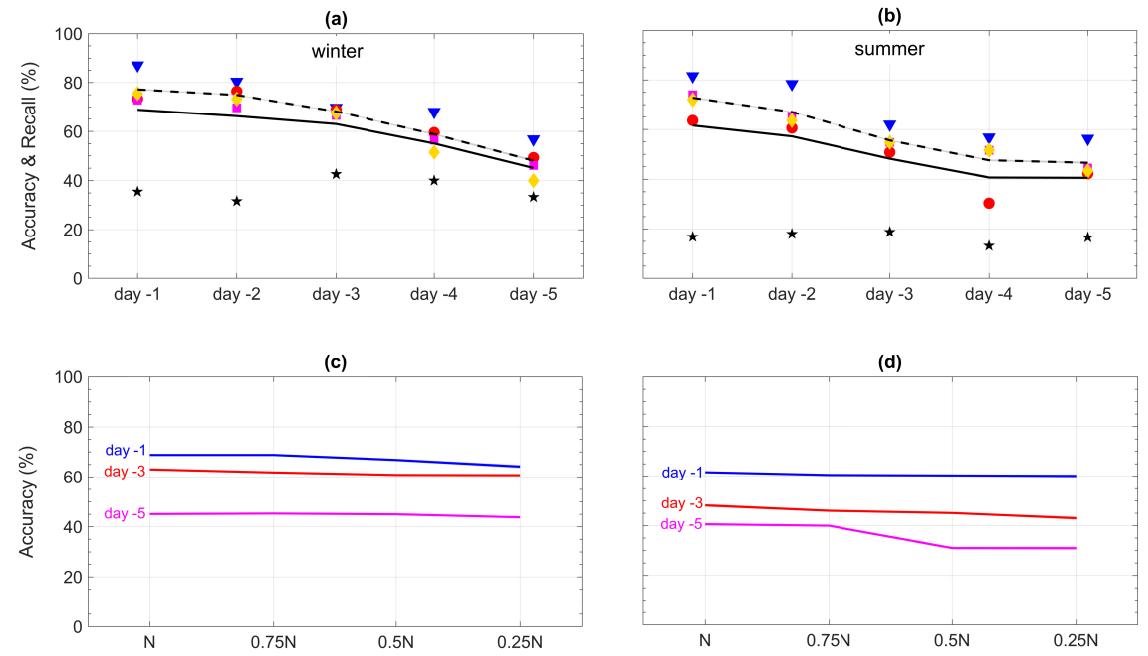

Figure 3: Performance of CapsNets in predicting heat waves and cold spells using Z500 patterns at various lead times. (a)-(b) The symbols show the accuracy at different lead times for each cluster: star (0), triangle (1), square (2), diamond (3), and circle (4). The solid (dashed) lines show the total accuracy (recall). (c)-(d) Total accuracy at prediction lead times 1, 3, and 5 days versus the size of the training set $(N=750$; fractions are rounded to the nearest integer if needed). Results in (a)-(b) are obtained with the largest training set. The symbols show the accuracy averaged over 3 randomly-drawn pairs of training/testing sets. The lines and their shading depict the mean and $\pm 1 \mathrm{SD}$ of accuracy or recall computed for the 3 pairs; the shadings are narrow, demonstrating the small SD and robustness of the results. 
This is a non peer-reviewed preprint submitted to EarthArxiv and is in revision in Geophysical Research Letters
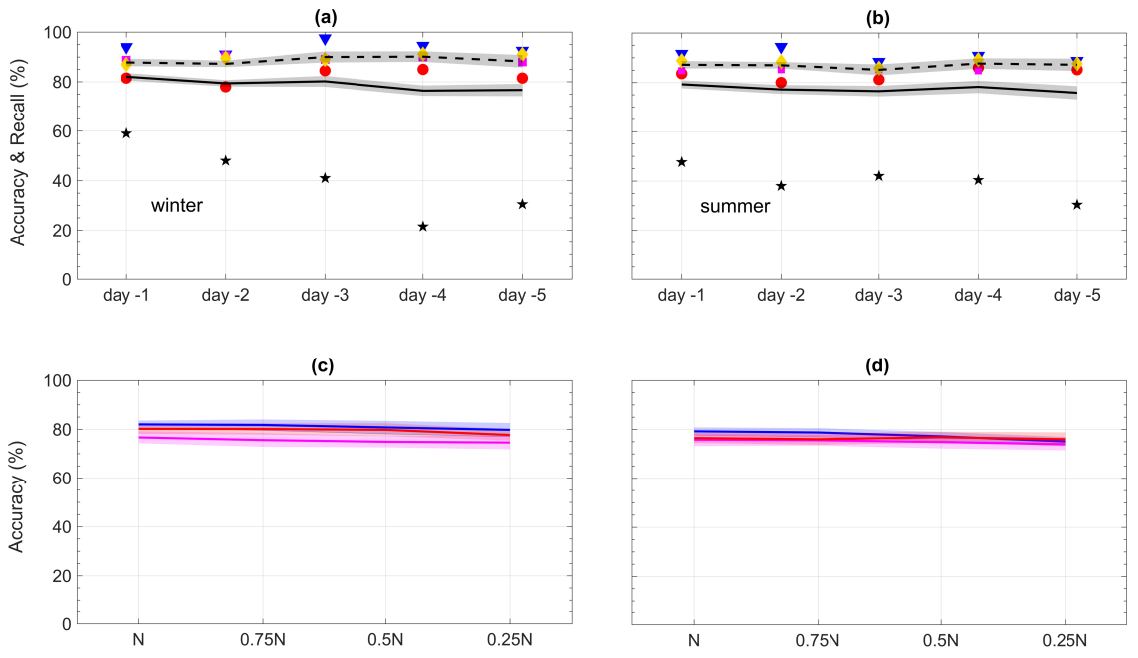

Figure 4: Same as Figure 3 but for the performance of CapsNets in predicting heat waves and cold spells using both T2m and Z500 patterns. The shadings show $\pm 1 \mathrm{SD}$; the SD values are higher in the multi-modal approach. In (c)-(d), the change of accuracies with the size of the training set is small for lead times of 1,3 , and 5 days (labels not shown). 